
\title{
R Reserarch Suare \\ The feasibility of bedside ultrasound in confirmation of Endotracheal tube position in neonates compared with CXR
}

\section{Nasrin Khalesi}

Iran University of Medical Sciences

Marzie Dehgan

Iran University of Medical Sciences

Elham Zarei ( $\square$ zarei.e@IUMS.ac.ir)

Iran University of Medical Sciences https://orcid.org/0000-0002-2130-405X

Research article

Keywords: Bedside Ultrasonography, Chest X-ray, Endotracheal intubation

Posted Date: October 30th, 2020

DOI: https://doi.org/10.21203/rs.3.rs-50177/v2

License: () (1) This work is licensed under a Creative Commons Attribution 4.0 International License.

Read Full License 


\section{Abstract}

\section{Background:}

Endotracheal intubation is a common procedure in the neonatal intensive care unit (NICU). Rapid and accurate confirmation of the endotracheal tube (ETT) position is critical. Routinely CXR is used for evaluation of ETT position that has some disadvantages. So there has been a considerable interest to find alternative modality with minimal complication and acceptable accuracy.

The purpose of our study is the evaluation of ultrasound efficacy in confirmation of ETT position in neonates comparison to CXR.

Methods: This cross-sectional study was conducted on 66 intubated neonates in NICU of Ali -Asghar children Hospital, between January 2018 and January 2019. To determine the ETT position, CXR and ultrasound were requested at the same time. Each neonate had a bedside ultrasound (US) with a highfrequency linear transducer. Accuracy, sensitivity, specificity, negative predicted value (NPV), positive predicted value (PPV) and Kappa agreement coefficient was used to compare the US and CXR results.

Results: Sixty-six intubated neonates included in this study had a mean gestational age of $34.98 \pm 3.7$ weeks and mean birth weight of $2284.7 \pm 915 \mathrm{~g}$. US images were taken a mean of $3 \pm 0.6 \mathrm{~h}$ after CXR. The ETT was visualized by the US in all newborns examined.US revealed a sensitivity of $88 \%(95 \% \mathrm{Cl}, 68.78 \%$ to $97.45 \%)$, specificity of $84 \%$ (95\% Cl, 63.92\% 95.46\%), PPV of $84.62 \%$ (95\% Cl, 68.89\% to $93.18 \%), \mathrm{NPV}$ of $87.50 \%$ (95\% Cl, $70.49 \%$ to $95.35 \%)$, diagnostic accuracy of $86 \%(95 \% \mathrm{Cl}, 73.26 \%$ to $94.18 \%)$, positive likelihood ratio of 5.50 (95\% Cl 2.21 to 13.66), and Negative Likelihood Ratio of 0.14 (95\% Cl 0.05 to 0.46 ) compared to chest radiography. Overall, the Kappa value showed very good agreement in confirming the correct position of the tracheal tube placement (Kappa coefficient 0.72, p-value <0.001). The mean time taken for confirmation of ETT position by US 4 minutes and by CXR was 20 minutes.

Conclusion: The findings of this study demonstrate ultrasound has acceptable accuracy for confirmation of ETT position in neonates and is a safer and faster method in comparison to CXR .

\section{Background}

Endotracheal intubation is a common procedure in the neonatal intensive care unit (NICU) $(1,2)$. Because malposition of ETT is a common finding in infants ( 3 ) and could be associated with various serious outcomes such as esophageal intubation, Rt upper lobe or left lung atelectasis and hypoxemia, pulmonary air leak syndrome, fast and accurate determination of ETT position is critical $(1,4)$ There are numerous methods for ETT position confirmation such as visualizing vocal cords during direct laryngoscopy auscultation, observation of chest movement and capnography ( 4 ).In most centers, CXR is routinely used after each intubation and reintubation for confirmation of ETT position . [9, 10]

Despite the reliability of CXR in detecting ETT position, this modality is associated with some disadvantages such as radiation exposure, inability to differentiate esophageal intubation from tracheal 
intubation, time-consuming, and need to reposition of sick neonates (5).

Regarding the mentioned disadvantages, there has been increasing interest to find an alternative method with similar accuracy and minimal complications.

The ultrasound could be done quickly and bedside without radiation exposure and causes no or minimal disturbance of the sick neonate position.

Multiple studies suggested that bedside ultrasound has good effectiveness for ETT position in adults, nevertheless, there is no consensus

In replacing CXR with bedside ultrasound for confirmation of the proper position of ETT in neonates.

Our aim in this study is evaluation of the efficacy of bedside ultrasound in the determination of ETT position in intubated neonates in NICU compared with CXR.

\section{Methods}

This study population included 66 neonates who were admitted to the NICU of Ali -Asghar children hospital and required intubation for respiratory distress between January 2018 and January 2019.

Neonates that ETT tip was not obviously seen by CXR and whose bedside ultrasound could not be done simultaneously with CXR were excluded from the study.

After each intubation or re-intubation, CXR was routinely obtained.

The appropriate position of ETT on CXR was defined as between thoracic inlet (C7-T1 disc space) and 10 $\mathrm{mm}$ above the carina (or T2 -T3 disc space).

If the ETT tip was located more than $10 \mathrm{~mm}$ from carina (or beyond the body of T3) or located above thoracic inlet (or C7-T1 disc space), it would be considered deep and high position respectively $(1,11,12$ )

After intubation ,ETT position was confirmed with clinical signs and oxygen saturation monitoring and CXR and ultrasound were requested at the same time.

The ultrasound was performed by Five trained radiologists were unaware of CXR results.

All radiologists have been trained in chest ultrasound for at least one month and airway ultrasound training for at least one hour by

an assistant professor of radiology that has seven years' experience in neonatal ultrasound .

The ultrasound was done with a high-frequency linear probe that placed superior to the suprasternal notch in axial and longitudinal axis and then in the parasternal region with the longitudinal axis ( for 
visualization aortic arch )with the neck slightly extended.

To better localization, gentle longitudinal movement of ETT about $2 \mathrm{~mm}$ in each direction was done.

When ETT is inside the trachea, axial ultrasound shows one air-mucosal interface with a reverberation artifact and posterior shadowing.

In the longitudinal view, ETT produces double continuous linear echogenicity immediately posterior to the tracheal rings with broken linear echogenicity appearance.

The distance between the ETT tip and maximal curvature of the aortic arch (AA) as a marker was measured

If ETT tip was between the lower level of thyroid and more than $10 \mathrm{~mm}$ from AA was defined as proper position, if it was above the lower level of thyroid or less than $10 \mathrm{~mm}$ above the apex of AA was classified as high and deep position respectively. $(7,8.9,11)$

The ETT was visualized in all subjects on ultrasound. There was no case of esophageal intubation.

The required time for evaluation of the ETT position by two modalities was recorded for each patient.

\section{Results}

A total of 66 intubated neonates enrolled in the study. The mean gestational age and birth weight of the neonates were $34.98 \pm 3.7$ weeks and $2284.7 \pm 915 \mathrm{~g}$ respectively. Of the neonates, $44(66.7 \%)$ were male and $22(33.3 \%)$ were female. The demographics and clinical characteristics of study subjects are presented in Table 1.

Table 1: The demographics and clinical characteristics of study subjects

\section{Characteristics}

Birth weight(g)

Gestational age(week)
Minimum Maximum $\begin{gathered}\text { Mean } \\ \pm S D\end{gathered}$ Median

$\begin{array}{llll}850 & 3820 & 34.98 & 2500\end{array}$

$\pm 3.7$

$2284.7 \quad 36$ $\pm 915$

Endotracheal tube position determined by CXR was proper in 56 (84.8\%), and improper in 10 (15.2\%) cases while endotracheal tube position as determined by Ultrasound was proper in 47 (71.21\%) cases, and improper in 19 (28.78\%) cases, and none showed esophageal intubation. 
Compared to CXR, US examination for detecting the proper position of ETT revealed a sensitivity, specificity, positive predictive value, negative predictive value, diagnostic accuracy, positive likelihood ratio, and negative likelihood ratio of $88 \%, 84 \%, 84.62 \%, 87.5 \%, 86 \%, 5.5 \%, 0.14 \%$ respectively. Accuracy of detecting the proper position of ETT by ultrasonography in comparison with CXR is presented in Table 2.

Table 2: Accuracy of detecting proper position of ETT by ultrasonography in
comparison with chest radiograph

\section{Index}

Sensitivity

Specificity

Positive predictive value

Negative predictive value

Diagnostic accuracy

Positive likelihood ratio

Negative likelihood ratio

Cl: Confidence Interval

\section{Value $(95 \% \mathrm{Cl})$}

$88 \%$ ( $68.78 \%$ to

$97.45 \%)$

$84 \%$ ( $63.92 \%$ to

$95.46 \%)$

$84.62 \%$ ( $68.89 \%$ to $93.18 \%)$

$87.5 \%$ (70.49\% to $95.35 \%)$

$86 \%(73.26 \%$ to

$94.18 \%)$

$5.5 \%(2.21 \%$ to

$13.66 \%)$

$0.14 \%(0.05 \%$ to

$0.42 \%)$

There was no complication during the bedside ultrasound in the studied neonates. The total accuracy of US in affirmation of ETT position based on the area under the receiver operating characteristic (ROC) curve was $86 \%$. Mean (SD) time interval between CXR and US evaluation was $3 \pm 0.6 \mathrm{~h}$. The median time required for CXR and US assessment for determination of ETT position was 20 minutes and 4 minutes respectively.

\section{Discussion}

Because the improper position of ETT is common and considered as one of the most important complications of ETT in neonates, the presence of quick and accurate method for localization of ETT tip is critical in neonates. Among various methods for evaluation of the ETT position, a reliable and commonly used modality is CXR. 
Nevertheless, CXR is associated with some disadvantages such as radiation exposure, inability to differentiate esophageal intubation from tracheal intubation, time-consuming, and need to reposition of sick neonates (5).

Ultrasound seems

to be a good alternative method for evaluation of ETT tip position regarding its potential advantages such as no radiation, portability and being fast, and with no or minimal disturbance of sick neonates position during the performance.

For the first time, ultrasound was used for evaluation of ETT position in two small trials in the 1980s (7, 8) that showed the ETT tip was reliably identified by ultrasound in neonates.

Since that time, various studies assessed the accuracy of bedside ultrasound to the determination of ETT position in comparison to CXR.

Most of them demonstrated no statistically significant differences between the US and CXR in identifying the correct ETT position $(2,9,10$, and 11) nevertheless de Kock et al. showed there was poor agreement between findings of CXR and US (12)

Our study results showed that bedside ultrasound had acceptable accuracy comparison to CXR for detecting the correct position of ETT in the neonate and also, was the faster and safer method than CXR with no complication during performance.

Despite agreement among various investigations about the accuracy of ultrasound, but there was no consensus about the method to find out the proper position of ETT by the US so that some authors were used aortic arch as ultrasound marker $(7,8,9,11)$ and some of them were used Rt pulmonary artery( 10,13).

Also about the definition of the high position of ETT on ultrasound, various markers were used.

Despite awareness of adverse outcomes of early radiation exposure, and regarding the accuracy of ultrasound, there is less acceptance in the use of ultrasound in neonatal intubation. (14)

Two major limitations of most studies were that ultrasound images were obtained by one or two highly skilled pediatric radiologist and the sample size were small.

$(7,8,9$ and 15$)$

Our study included 66 intubated neonates that are the one of largest investigations of its kind to date, also we used a larger number of radiologists without previous skill in tracheal ultrasound that was trained for one hour by an attending pediatric radiologist which increase the generalizability of this technique.

\section{Conclusion}


Regarding multiple advantages of ultrasound and its good accuracy in determination of ETT tube position we recommended with training neonatologist, could replace CXR with ultrasound for evaluation ETT position and avoid unnecessary radiation exposure.

\section{Declarations}

\section{Ethic approval and consent to participate}

The informed consent was verbally confirmed by all participants and the Ethics committee of Iran University of Medical Sciences confirmed all procedures of this study (IR.IUMS.FMD.REC.1397.257).

Prior to ultrasound, written informed consent was obtained from all parents subjects.

\section{Consent to publish}

The authors declare that they consent to publish

\section{Availability of data and materials}

The datasets used and/or analyzed during the current study are available from the corresponding author on reasonable request.

\section{Competing interest}

No competing interest

\section{Funding}

No funding

\section{Author's Contributions}

EZ (study conception \& design and Critical revision), NK and MD (Acquisition of data, Analysis and interpretation of data \& Drafting of manuscript). All authors have read and approved the manuscript.

\section{Acknowledgement}

The Authors would like to thank Ali Asghar Clinical Research Development Center (AACRDC), Iran University of Medical Sciences (IUMS) for their support to perform this project.

\section{Authors' information}

Nasrin Khalesi 
Department of Neonatology, Ali Asghar Hospital, Iran University of Medical Sciences (IUMS), Tehran, Iran nasrinkhalessi@yahoo.com

Marzie Dehgan

Department of Neonatology, Ali Asghar Hospital, Iran University of Medical Sciences (IUMS), Tehran, Iran

marzi.dehgan@gmail.com

Elham Zarei

Department of Radiology, Ali Asghar Children Hospital, Iran University of Medical Sciences, Tehran, Iran

Email: zarei.e@iums.ac.ir

ORCHID: https://orcid.org/0000-0002-2130-405X

Corresponding Author: Elham Zarei

Department of Radiology, Ali Asghar Children Hospital, Iran University of Medical Sciences, Tehran, Iran. Email: zarei.e@iums.ac.ir

ORCHID: https://orcid.org/0000-0002-2130-405X

Telephone: +989197377864

\section{Abbreviations}

NICU: neonatal intensive care unit

ETT: endotracheal tube

US: ultrasound

\section{References}

1. Deepak Sharma, Seyyed Ahmad Tabatabaii \& Nazanin Farahbakhsh (2019) Role of ultrasound in confirmation of endotracheal tube in neonates: a review, The Journal of Maternal-Fetal \& Neonatal Medicine, 32:8, 1359-1367, DOI: 10.1080/14767058.2017.1403581

2. Dennington, D., Vali, P., Finer, N. N., \& Kim, J. H. (2012). Ultrasound Confirmation of Endotracheal Tube Position in Neonates. Neonatology, 102(3), 185-189. doi:10.1159/000338585

3. Harris EA, Arheart KL, Penning DH. Endotracheal tube malposition within the pediatric population: a common event despite clinical evidence of correct placement. Can J Anesth. 2008;55(10):685-690. 
4. Alonso Quintela P, et al. Usefulness of bedside ultrasound compared to capnography and X-ray for tracheal intubation. An Pediatr (Barc). 2014;81:283-288.

5. Vijaykumar R, Saboo AR (2014) Review of Different Methods Used for Confirmation of Endotracheal Tube Placement in Newborns. J Neonatal Biol 3: 154. doi:10.4172/2167-0897.1000154

6. Das SK, Choupoo NS, Haldar R, et al. Transtracheal ultrasound for verification of endotracheal tube placement: a systematic review and meta-analysis. Can J Anesth. 2015;62(4):413-423.

7. Slovis TL, Poland RL. Endotracheal tubes in neonates: sonographic positioning. Radiology 1986; 160(1): 262-263.

8. Lingle PA. Sonographic verification of endotracheal tube position in neonates: a modified technique. J Clin Ultrasound 1988; 16(8): 605-609.

9. Saul D, Ajayi S, Schutzman DL, et al. Sonography for a complete evaluation of neonatal intensive care unit central support devices: a pilot study. J Ultrasound Med. 2016;35(7):1465-1473.

10. Najib K, Pishva N, Amoozegar H, et al. Ultrasonographic confirmation of endotracheal tube position in neonates. Indian Pediatr. 2016;53(10): 886-888.

11. Chowdhry R, Dangman B, Pinheiro JM. The concordance of ultrasound technique versus $X$-ray to confirm endotracheal tube position in neonates. J Perinatol. 2015;35(7):481-484.

12. De Kock SH, Otto SF, Joubert G. The feasibility of determining the position of an endotracheal tube in neonates by using bedside ultrasonography compared with chest radiographs. S Afr J Child Health. 2015; 9(1):3-5.

13. Dennington $D$, Vali $P$, Finer $N N$, et al. Ultrasound confirmation of endotracheal tube position in neonates. Neonatology. 2012;102(3):185-189.

14. Scott MV, Fujii AM, Behrman RH, Dillon JE. Diagnostic ionizing radiation exposure in premature patients. J Perinatol 2014; 34(5): 392-395.

15. Dennington D, Vali P, Finer NN, Kim JH: Ultrasound confirmation of endotracheal tube position in neonates. Neonatology 2012,102(3):185-189. 10.1159/000338585 